\title{
ЭФФЕКТ УСТРАНЕНИЯ ДЕФИЦИТА ВИТАМИНА D НА РИСК ИНФИЦИРОВАНИЯ COVID-19 У МЕДИЦИНСКИХ РАБОТНИКОВ ИНФЕКЦИОННОГО СТАЦИОНАРА
}

Каронова Т.Л., Быкова Е.С., Андреева А.Т., Гринева Е.Н.

Исследования последних лет продемонстрировали иммуномодулирующие свойства витамина D и обозначили возможность его применения для профилактики развития и уменьшения степени тяжести течения острых респираторных вирусных заболеваний. В период пандемии новой коронавирусной инфекции препараты витамина D вошли в протоколы ведения больных COVID-19. Целью исследования, проведенного в Центре Алмазова, было оценить эффективность применения витамина D у медицинских работников инфекционного стационара для снижения риска инфицированности и степени тяжести инфекционного процесса. Результаты исследования показали высокую распространенность недостатка и дефицита витамина D у медицинских работников и достижение нормального уровня 25(OH)D в сыворотке крови на фоне приема 100000 ME в течение двух недель. Последующий прием колекациферола в дозе $5000 \mathrm{ME}$ в сутки сопровождался нормализацией уровня обеспеченности витамином $D$ в 50\% случаев, в то время как только четверть включенных в исследование лиц имела нормальный уровень 25(OH)D крови на. фоне суточного приема дозы 2000 ME. Прием колекациферола не был ассоциирован со снижением риска инфицированности SARS-CoV-2 у медицинских работников инфекционного стационара, однако большинство случаев заболевания, выявленных к концу трех месяцев наблюдения, протекало бессимптомно 